\title{
Review
}

\section{The diagnosis and management of pre-invasive breast disease Pathology of atypical lobular hyperplasia and lobular carcinoma in situ}

\author{
Peter T Simpson ${ }^{1}$, Theodora Gale 1 , Laura G Fulford ${ }^{1}, 2$, Jorge S Reis-Filho ${ }^{1}$ and Sunil R Lakhani ${ }^{1,3}$ \\ ${ }^{1}$ The Breakthrough Toby Robins Breast Cancer Research Centre, Institute of Cancer Research, London, UK \\ ${ }^{2}$ The Ludwig Institute for Cancer Research, London, UK \\ ${ }^{3}$ The Royal Marsden Hospital, London, UK \\ Corresponding author: Sunil R Lakhani (e-mail: Sunil.Lakhani@icr.ac.uk)
}

Published: 29 July 2003

Breast Cancer Res 2003, 5:258-262 (DOI 10.1186/bcr624)

(c) 2003 BioMed Central Ltd (Print ISSN 1465-5411; Online ISSN 1465-542X)

\begin{abstract}
The term lobular neoplasia refers to a spectrum of lesions featuring atypical lobular hyperplasia and lobular carcinoma in situ (LCIS). The histopathological characteristics of these lesions are well documented. What is less well understood is the management implications of a patient diagnosed with LCIS; treatment regimes vary and are somewhat controversial. LCIS is now considered a risk factor and a non-obligate precursor for the subsequent development of invasive cancer.
\end{abstract}

Keywords: atypical lobular hyperplasia, breast cancer, lobular carcinoma in situ, lobular neoplasia, precursor lesion

\section{Introduction}

Atypical lobular hyperplasia (ALH) and lobular carcinoma in situ (LCIS) - lesions that are also referred to under the umbrella heading of 'lobular neoplasia' (LN) - occur relatively infrequently in the breast. However, problems and controversies surrounding the most appropriate terminology and classification for these lesions, and the best course of long-term management after diagnosis, are far from infrequent.

Foote and Stewart first coined the term LCIS in 1941 [1], choosing the name to highlight the morphological similarities between the cells of LCIS and those of frankly invasive lobular carcinoma. They recognised parallels with ductal carcinoma in situ (DCIS), namely foci of neoplastic cells that were still contained within a basement membrane. In anticipating that LCIS, like DCIS, was a step along the pathway to invasive cancer, they recommended mastectomy as the standard form of treatment; this management plan was adopted for many years. The term ALH was subsequently introduced to describe morphologically similar but less well developed lesions. LN was a term introduced by Haagensen in 1978 [2] to cover the full range of proliferation, including both ALH and LCIS within the spectrum.

ALH and LCIS have since become well-established histopathological entities in the classification of breast neoplasia, but it has become clear over the past 60 years that they are not precursor lesions for invasive carcinoma in the same way as high-grade DCIS of comedo type [3-6]. A diagnosis of ALH/LCIS today is often seen as a 'risk indicator' for subsequent carcinoma rather than a true precursor. Radical surgical treatment has fallen out of favour but there is a lack of consensus on what the most appropriate management of patients diagnosed with ALH/LCIS should be. Recommendations for treatment vary from follow-up with regular mammography, to followup alone or simply 'no action' $[2,7,8]$. However, recent work is once again suggesting that LCIS is indeed a nonobligate precursor lesion for carcinoma, a finding that might have significant implications for the management of patients diagnosed with this disease. 


\section{Epidemiology of LN}

LCIS is most frequently diagnosed in women aged between 40 and 50 years (less than 10\% of patients with LCIS are postmenopausal), which is a decade earlier than the age of women diagnosed with DCIS. Estimating the incidence of LCIS is fraught with difficulty. There are no specific clinical abnormalities, in particular no palpable lump, and LCIS is only rarely visible on mammography when an uncommon calcifying subtype is present $[9,10]$. When examining a pathological specimen, there are no macroscopic features characteristic of LCIS. The diagnosis of LCIS is therefore usually an incidental finding in breast biopsy performed for other indications. For these reasons the true incidence of LCIS in the general population is unknown, and many asymptomatic women presumably go unnoticed. The incidence of LCIS in otherwise benign breast biopsy is between $0.5 \%$ and $3.8 \%[2,11]$.

Characteristically, LCIS is multifocal and bilateral in a large proportion of cases. Over 50\% of patients diagnosed with $\mathrm{LN}$ contain multiple foci in the ipsilateral breast and about $30 \%$ of cases will have further LCIS in the contralateral breast [12-14]. This multifocality, in a clinically undetectable lesion, is one of the reasons why planning subsequent management is so difficult.

\section{Histological features of LN}

The criteria for the histological diagnosis of ALH and LCIS are well established. LCIS is composed of a monomorphic population of usually small, round, polygonal or cuboidal cells, with a thin rim of clear cytoplasm and a high nuclearcytoplasmic ratio (Fig. 1). Cells containing clear vacuoles, known as intracytoplasmic lumina or magenta bodies, are often seen, and when they are identified in a fine needle aspirate from the breast, they strongly suggest the presence of a lobular lesion (including ALH, LCIS and invasive lobular carcinoma). The cells are loosely cohesive, regularly spaced, and fill and distend the acini; however, overall lobular architecture is maintained. Glandular lumina are not seen, and mitoses, calcification and necrosis are uncommon. Pagetoid spread, in which the neoplastic cells extend along adjacent ducts, between intact overlying epithelium and underlying basement membrane, is also frequently seen.

The cells of classic LCIS, as described above, can also be referred to as type A cells. Type B cells are a well-recognised subtype of LCIS cells, with mildly to moderately larger nuclei showing some increase in pleomorphism. A more recently described entity is that of pleomorphic LCIS (PLCIS). The cells in this lesion show more marked pleomorphism and distinctly larger nuclei with nucleoli. Central necrosis and calcification within lobules are features of note. In a situation analogous to ALH versus LCIS, there might be some difficulty in terminology and practical differentiation between a case of LCIS with type B cells and

\section{Figure 1}

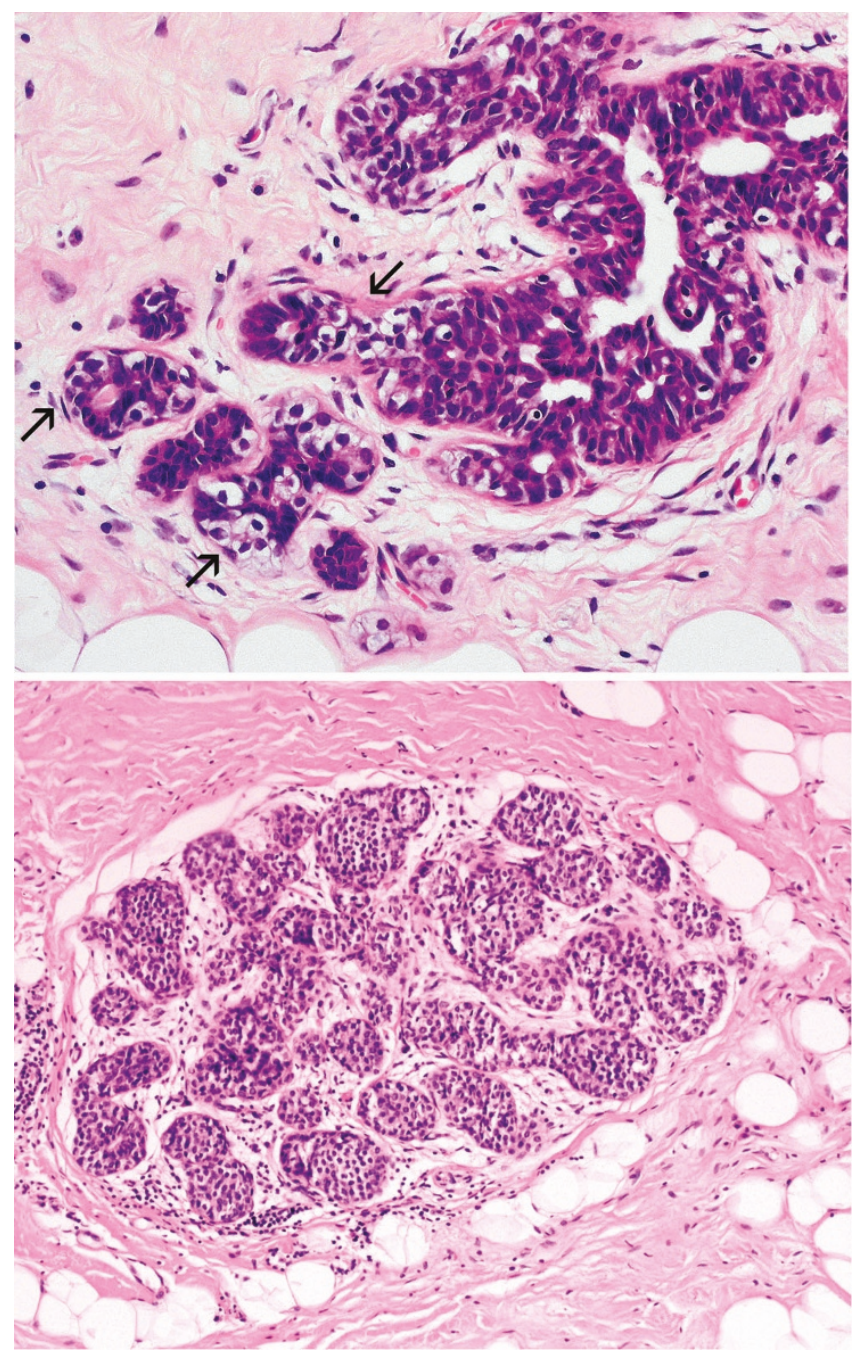

Differentiation of atypical lobular hyperplasia from lobular carcinoma in situ is based on the extent of proliferation and the distension of the lobular unit. In this case of atypical lobular hyperplasia (upper panel), all acini are filled with neoplastic lobular type A cells (arrows), yet very few are distorted. In contrast, the lower panel demonstrates that more than $50 \%$ of acini are filled and distended, indicating a diagnosis of lobular carcinoma in situ. Haematoxylin/eosin stain.

that of PLCIS. Sneige and colleagues [15] have described type $B$ cells as containing nuclei that are up to double the size of a lymphocyte (type A cells are 1-1.5 times larger), whereas PLCIS nuclei are typically four times larger. These subtypes might represent a spectrum of lesions, but it is possible that PLCIS has different biological behaviour and implications from those of classic LCIS. It is therefore important to recognise and document the presence of this variant.

For a diagnosis of LCIS, more than half the acini in an involved lobular unit must be filled and distended by the characteristic cells, leaving no central lumina (Fig.1). For 
practical diagnostic purposes, distension translates as eight or more cells present across the diameter of an acinus. A lesion is regarded as ALH when it is less well developed and less extensive than this, for instance when the characteristic cells only partly fill the acini, with no or only mild distension of the lobule (Fig.1). Lumina might still be visible and the number of acini involved is less than half. Myoepithelial cells can be seen admixed with the neoplastic population.

Clearly the differentiation between ALH and LCIS on these criteria is somewhat arbitrary, and associated with inter-observer and intra-observer variability. The use of the term LN to encompass the whole range of changes might therefore be preferable for diagnostic purposes. So far the term has not gained widespread use among pathologists. As discussed below, the justification for continuing to use the ALH/LCIS terminology is that ALH has been shown to have a lower risk than LCIS of subsequent invasive carcinoma $[11,16,17]$.

A further system for classification of these lesions has been proposed by using the terminology 'lobular intraepithelial neoplasia' (LIN) and with subdivision, based on morphological criteria and clinical outcome, into three grades: LIN 1, LIN 2 and LIN 3 [18]. The assertion is that the risk of subsequent invasive carcinoma is related to increasing grade of LIN; however, there is as yet no consensus of opinion, and data to support this view are preliminary. In view of the rapid evolution in technology (see the review on new technology in this series [19]), the classification systems are likely to undergo further change as molecular data are incorporated. Hence, at present, it does not seem prudent to introduce yet another interim classification. We should take a lesson from the multiple lymphoma classifications that led to considerable confusion in patient management.

\section{Differential diagnosis of LN}

Occasional diagnostic difficulty can occur in cases in which poor tissue preservation leads to an artefactual appearance of discohesive cells within a lobular unit, resulting in an overdiagnosis of LCIS. Another well-recognised problem occurs when LCIS is superimposed on a type of benign breast lesion known as sclerosing adenosis, which causes a distortion of lobular units and a sclerotic stroma. The combination of abnormal architecture and the proliferative lobular cells can easily be mistaken for an invasive carcinoma by the unwary. In this situation, immunohistochemistry to demonstrate the myoepithelial cell layer or basement membrane can be useful in making the distinction.

The most important, and the most difficult, differential diagnosis of LCIS is from low-nuclear-grade, solid DCIS. This entity carries wholly different management implications for the patient because it usually requires surgical excision, whereas arguably, as discussed, LCIS might warrant no further action. Correct identification is therefore essential. However, distinction of LCIS from low-grade solid DCIS can be very difficult because morphologically they can be strikingly similar (Fig. 2), especially when DCIS involves the acini with minimal or no lobular distortion. The presence of secondary lumen formation and cellular cohesion might point to a ductal lesion rather than LCIS. Immunohistochemical analysis of the lesion can prove useful because E-cadherin, a cell membrane molecule involved in cell adhesion, is typically absent in ALH/LCIS but present in DCIS (see the review on molecular genetics in this series [20]). In addition the expression of high-molecular-mass cytokeratin (CK34ßE12) is usually seen in LCIS but not in DCIS [21]. Occasionally, lesions show a combination of markers, suggesting that LCIS and low-grade solid DCIS can coexist within the same duct-lobular unit. In these circumstances, differentiation between the two is often not possible and both diagnoses should be given.

\section{Implications of LN}

Although it is clear that LCIS is not an obligate precursor to invasive lobular carcinoma, many studies have shown that a proportion of women with LCIS go on to develop invasive carcinoma, with a risk of 6.9 times to about 12 times that of women without LCIS $[2,22]$.

Page and colleagues $[11,16]$ reported that the relative risk of subsequently developing breast cancer was different in patients diagnosed with ALH compared with LCIS. Patients diagnosed with ALH have a risk of 4-5 times that of the general population (namely women, of comparable age, who have had a breast biopsy performed with no diagnosis of proliferative disease) $[16,17]$. This relative risk is doubled to $8-10$ times for LCIS [11]. Thus, although LN is a helpful term for describing these lesions collectively, classification into ALH and LCIS might still be justified, or preferable for risk stratification and management decisions.

Data accumulated from nine separate studies revealed that $15 \%$ of 172 patients diagnosed with LCIS developed invasive carcinoma in the ipsilateral breast, and $9.3 \%$ of 204 patients developed invasive carcinoma in the contralateral breast [23]. The development of contralateral breast cancer is three times more likely in patients diagnosed with LCIS than without LCIS [24]. The risk of developing breast cancer is therefore also bilateral [12]. Reports have suggested that this risk is equal to both breasts; however, corroborating studies demonstrate that carcinoma is three times more likely to develop in the ipsilateral relative to the contralateral breast $[16,25,26]$.

The time taken to develop invasive cancer after diagnosis of LCIS is unclear. Page and colleagues [11] reported that 


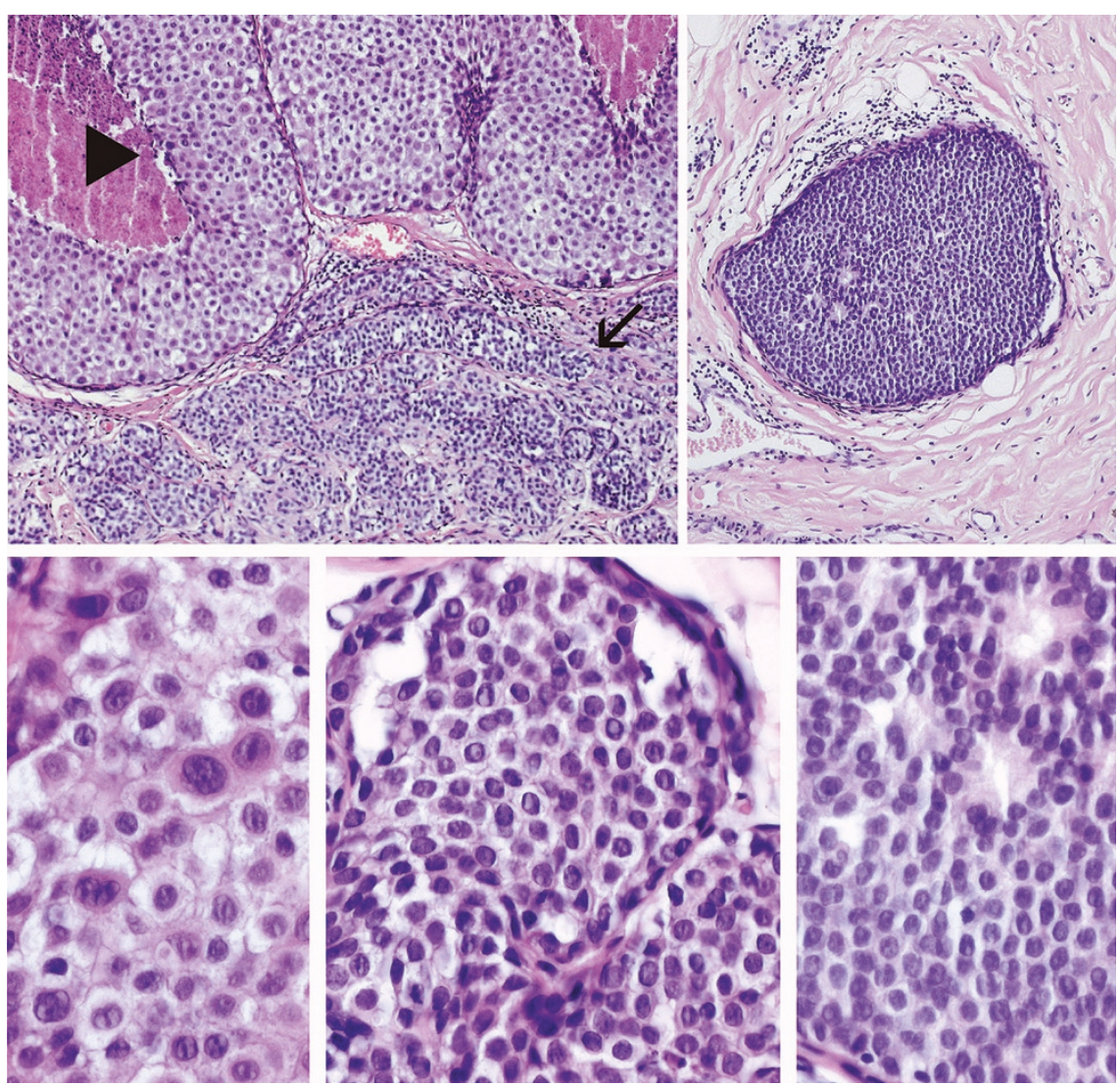

Differential diagnosis is often difficult between lobular carcinoma in situ (arrow in upper left panel) and low-nuclear-grade, solid ductal carcinoma in situ (upper right panel). Both lesions exhibit characteristic small monomorphic cells with a high nuclear-cytoplasmic ratio (high-power views, lower middle and lower right panels, respectively). In contrast, high-grade ductal carcinoma in situ (arrowhead in upper left panel; high-power view, lower left panel) exhibits markedly different histopathological features, notably the cohesiveness of neoplastic cells, pleomorphic nuclei and abundant eosinophilic-to-amphiphilic cytoplasm. Haematoxylin/eosin stain.

two-thirds of women who developed invasive cancer did so within 15 years of biopsy, yet in a separate study over $50 \%$ of cases who developed cancer did so between 15 and 30 years after biopsy, with an average interval of 20.4 years [27].

Both invasive ductal carcinoma and invasive lobular carcinoma occur with LCIS. The coexistence of DCIS and LCIS might explain the invasive ductal carcinoma component observed, by which DCIS and not LCIS is the likely precursor lesion $[28,29]$. Evidence for the role of LCIS as a precursor for invasive lobular carcinoma is supported by the epidemiological data outlined above, the morphological similarity between cells of ALH/LCIS and lobular carcinoma and the development of tumours in regions localised to ALH/LCIS. Work on molecular aspects of lobular lesions, in particular that focusing on the marker E-cadherin, add to this view (see the review on molecular genetics in this series [20]).
Thus, the evidence, that $10-20 \%$ of patients identified with LCIS develop breast carcinoma in the 15-25 years after initial diagnosis, is compelling. Identifying this subgroup of individuals is not easy by current clinical or morphological means, although both morphological classifications and the use of E-cadherin have been suggested. Clearly further characterisation of these small lesions is necessary to disentangle the current problems faced in classification and management. It is hoped that

This article is the third in a review series on The diagnosis and management of pre-invasive breast disease - current challenges, future hopes, edited by Sunil R Lakhani.

Other articles in the series can be found at http://breast-cancer-research.com/articles/reviewseries.asp?series $=$ bcr_Thediagnosis 
the application of microdissection techniques and recently developed molecular technology will hold the key to our future understanding of LN.

\section{Competing interests}

None declared.

\section{References}

1. Foote FW Jr, Stewart FW: Lobular carcinoma in situ. A rare form of mammary cancer. Am J Pathol 1941, 17:491-496.

2. Haagensen $C D$, Lane N, Lattes R, Bodian C: Lobular neoplasia (so-called lobular carcinoma in situ) of the breast. Cancer 1978, 42:737-769.

3. Goldstein NS, Vicini FA, Kestin LL, Thomas M: Differences in the pathologic features of ductal carcinoma in situ of the breast based on patient age. Cancer 2000, 88:2553-2560.

4. Ringberg A, Idvall I, Ferno M, Anderson H, Anagnostaki L, Boiesen $\mathrm{P}$, Bondesson L, Holm E, Johansson S, Lindholm K, Ljungberg O, Ostberg G: Ipsilateral local recurrence in relation to therapy and morphological characteristics in patients with ductal carcinoma in situ of the breast. Eur J Surg Oncol 2000, 26:444451.

5. Silverstein MJ, Poller DN, Waisman JR, Colburn WJ, Barth A, Gierson ED, Lewinsky B, Gamagami P, Slamon DJ: Prognostic classification of breast ductal carcinoma-in-situ. Lancet 1995, 345:1154-1157.

6. Skinner KA, Silverstein MJ: The management of ductal carcinoma in situ of the breast. Endocr Relat Cancer 2001, 8:33-45.

7. Wheeler JE, Enterline HT, Roseman JM, Tomasulo JP, Mcllvaine $\mathrm{CH}$, Fitts WT, Jr., Kirshenbaum J: Lobular carcinoma in situ of the breast. Long-term followup. Cancer 1974, 34:554-563.

8. Wheeler JE, Enterline HT: Lobular carcinoma of the breast in situ and infiltrating. Pathol Annu 1976, 11:161-188.

9. Sapino A, Frigerio A, Peterse JL, Arisio R, Coluccia C, Bussolati G: Mammographically detected in situ lobular carcinomas of the breast. Virchows Arch 2000, 436:421-430.

10. Georgian-Smith D, Lawton TJ: Calcifications of lobular carcinoma in situ of the breast: radiologic-pathologic correlation. AJR Am J Roentgenol 2001, 176:1255-1259.

11. Page DL, Kidd TJ, Dupont WD, Simpson JF, Rogers LW: Lobular neoplasia of the breast: higher risk for subsequent invasive cancer predicted by more extensive disease. Hum Pathol 1991, 22:1232-1239.

12. Urban JA: Bilaterality of cancer of the breast. Biopsy of the opposite breast. Cancer 1967, 20:1867-1870.

13. Rosen PP, Senie R, Schottenfeld D, Ashikari R: Noninvasive breast carcinoma: frequency of unsuspected invasion and implications for treatment. Ann Surg 1979, 189:377-382.

14. Rosen PP, Braun DW Jr, Lyngholm B, Urban JA, Kinne DW: Lobular carcinoma in situ of the breast: preliminary results of treatment by ipsilateral mastectomy and contralateral breast biopsy. Cancer 1981, 47:813-819.

15. Sneige N, Wang J, Baker BA, Krishnamurthy S, Middleton LP: Clinical, histopathologic, and biologic features of pleomorphic lobular (ductal-lobular) carcinoma in situ of the breast: a report of 24 cases. Mod Pathol 2002, 15:1044-1050.

16. Page DL, Dupont WD, Rogers LW, Rados MS: Atypical hyperplastic lesions of the female breast. A long-term follow-up study. Cancer 1985, 55:2698-2708.

17. Dupont WD, Page DL: Risk factors for breast cancer in women with proliferative breast disease. N Engl J Med 1985, 312:146151.

18. Tavassoli FA: Pathology of the breast, edn 2. Norwalk, CT: Appleton and Lange; 1999.

19. Jeffrey SS, Pollack JR: The diagnosis and management of preinvasive breast disease: The promise of new technology in understanding pre-invasive lesions. Breast Cancer Res 2003, 5: in press.

20. Reis-Filho JS, Lakhani SR: The diagnosis and management of pre-invasive breast disease: Genetic alterations in preinvasive lesions. Breast Cancer Res 2003, 5: in press.

21. Bratthauer GL, Moinfar F, Stamatakos MD, Mezzetti TP, Shekitka KM, Man YG, Tavassoli FA: Combined E-cadherin and high molecular weight cytokeratin immunoprofile differentiates lobular, ductal, and hybrid mammary intraepithelial neoplasias. Hum Pathol 2002, 33:620-627.

22. Andersen JA: Lobular carcinoma in situ. A long-term follow-up in 52 cases. Acta Pathol Microbiol Scand [A] 1974, 82:519-533.

23. Andersen JA: Lobular carcinoma in situ of the breast. An approach to rational treatment. Cancer 1977, 39:2597-2602.

24. Haagensen CD, Lane N, Bodian C: Coexisting lobular neoplasia and carcinoma of the breast. Cancer 1983, 51:1468-1482.

25. Marshall LM, Hunter DJ, Connolly JL, Schnitt SJ, Byrne C, London SJ, Colditz GA: Risk of breast cancer associated with atypical hyperplasia of lobular and ductal types. Cancer Epidemiol Biomarkers Prev 1997, 6:297-301.

26. Page DL, Schuyler PA, Dupont WD, Jensen RA, Plummer WD Jr, Simpson JF: Atypical lobular hyperplasia as a unilateral predictor of breast cancer risk: a retrospective cohort study. Lancet 2003, 361:125-129.

27. Rosen PP, Kosloff C, Lieberman PH, Adair F, Braun DW Jr: Lobular carcinoma in situ of the breast. Detailed analysis of 99 patients with average follow-up of 24 years. Am J Surg Pathol 1978, 2:225-251.

28. Maluf $\mathrm{H}$, Koerner F: Lobular carcinoma in situ and infiltrating ductal carcinoma: frequent presence of DCIS as a precursor lesion. Int J Surg Pathol 2001, 9:127-131.

29. Rosen PP: Coexistent lobular carcinoma in situ and intraductal carcinoma in a single lobular-duct unit. $\mathrm{Am} J$ Surg Pathol 1980, 4:241-246.

\section{Correspondence}

Sunil R Lakhani, The Breakthrough Toby Robins Breast Cancer Research Centre, Institute of Cancer Research, London SW3 6JB, UK. Tel: +44 207153 5167; fax: +44 207153 5533; e-mail: Sunil. Lakhani@icr.ac.uk

mecular weight cytokeratin immunoprofile differentiates 